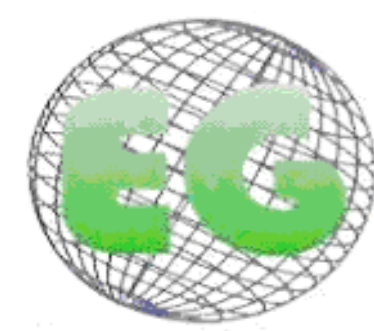

ISSN 1695-6141

$\mathrm{N}^{\circ} 23$
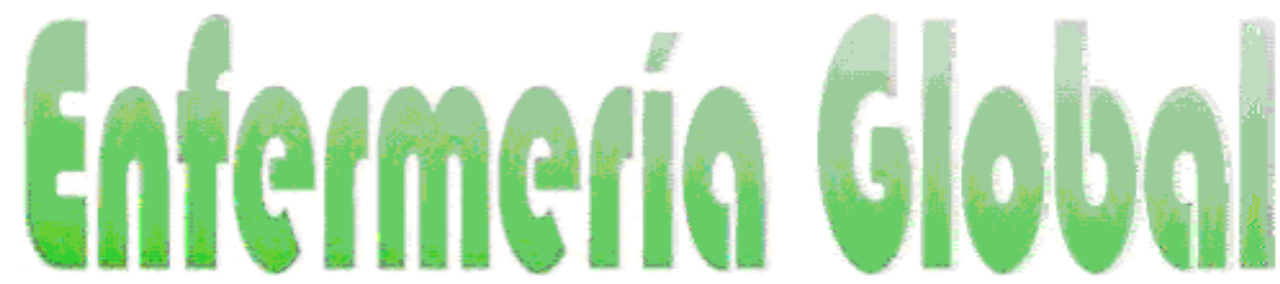

Revista electrónica trimestral de Enfermería

Julio 2011

www.um.es/egloball

\title{
Construcciones subjetivas sobre la Violencia de Género. Aportes para la prevención del problema.
}

Sulbjective constructions of gender violence. Contributions to the prevention of the problem.

\begin{abstract}
${ }^{*}$ Medina Maldonado, VE ** Camacaro Cuevas, MC ${ }^{* * *}$ Torres Torres, LM.
*Estudiante de Doctorado en la Universidad Martin Luther Halle-Wittenberg (Alemania) / Docente Escuela Enfermería " Dra. Gladys Román de Cisneros" UC, Facultad de Ciencias de la Salud.**Doctora en Ciencias Sociales / Coordinadora de la Unidad de Investigación para Estudios de Género "Bellacarla Jirón Camacaro" . UC, Facultad de Ciencias de la Salud, Sede Aragua. ${ }^{* * *}$ Magister en Enfermería. / Docente Escuela de Enfermería “ Dra. Gladys Román de Cisneros” UC, Facultad de Ciencias de la Salud, Carabobo-
\end{abstract} Venezuela.

Palabras clave: Calidad de vida; Violencia de género; Prevención primaria. Keywords: Quality of Life; Violence against Women; Primary Prevention.

\section{RESUMEN}

El reconocimiento de la Violencia de Género como un problema de Salud Pública compromete al sector sanitario a tomar medidas para su abordaje. Entendiendo la complejidad y variabilidad del problema la presente investigación tuvo como objetivo: Rastrear las subjetividades construidas por mujeres y hombres sobre la Violencia de Género a fin de promover iniciativas preventivas que contribuyan a elevar la calidad de vida de las mujeres. Es una investigación con enfoque de género abordada desde la modalidad cualitativa, las técnicas para la recolección de datos fueron la observación directa y el grupo focal de discusión. En la sistematización de los datos se manejó Atlas ti 6.2 (versión Demo) y para el razonamiento se uso análisis del discurso. Los resultados arrojaron: naturalización del problema, doble victimización, consentimiento de conductas patriarcales, censura de las mujeres que no exhiben comportamientos acordes con el rol femenino tradicional y recomendaciones para la prevención de la Violencia de Género.

\section{ABSTRACT}

The recognition of gender violence as a public health problem in the health sector supposes taking steps to address it. Understanding the complexity and variability of the problem, this research aimed to: Track the subjectivities built by women and men about gender violence to promote prevention initiatives that contribute to a better quality of life of women. It is a gender-sensitive research approached from the qualitative method. Techniques for data collection were the direct observation 
and focus group discussions. The systematization of the data is handled by SSPS and use reasoning and discourse analysis. The results showed: naturalization of the problem, double victimization behaviors consent, patriarchal censorship of women who do not exhibit behavior consistent with the traditional female role and recommendations for the prevention of gender violence.

\section{INTRODUCCIÓN}

El género se considera como una constante construcción de la identidad de mujeres y varones, corresponde a elementos más complejos que la simple asignación de roles por la disgregación biológica. Al respecto, Butler, J en Bonder (1998) mantiene que "El sexo no es lo que uno es sino en lo que uno se convierte. Por lo demás, el dualismo de lo biológico y lo cultural, no sería más que otra expresión de una lógica binaria que funda y legitima ordenamientos jerárquicos al oponer hombre y mujer, cuerpo y espíritu o psique, razón y emoción, etc."[1]. Desde esta corriente, es posible aseverar que la perspectiva de género es una categoría de análisis que examina y reflexiona sobre la subjetividad de mujeres y varones en diferentes sociedades y momentos históricos, sus posiciones en las relaciones de poder, en el acceso a los servicios o beneficios y las repercusiones de dichos factores durante su desenvolvimiento en la vida cotidiana.

Indiscutiblemente, que el desequilibrio existente en lo relacionado con el acceso a la información, el ejercicio del poder, la obtención de recursos sociales, la toma de decisiones, responsabilidades y expectativas origina lo que conocemos como inequidad de género, aspecto que condiciona la salud y la calidad de vida de mujeres y varones. Allí radica la importancia de incluir está perspectiva en el sector sanitario para identificar su impacto en el proceso salud-enfermedad y así evitar las diferencias que pueden perjudicar sistemáticamente a los(as) integrantes de una sociedad. Sin embargo, para lograr este ideal, el sector sanitario deberá tener en cuenta el significado que otorga la comunidad en general a la actividad femenina y masculina para aportar de manera conjunta medidas que permitan superar las desigualdades existentes.

Uno de los problemas más graves que siguen enfrentando en la actualidad muchas mujeres del mundo y que deterioran de manera categórica su salud física y mental es el relacionado con la Violencia que para los fines de este estudio se manejará el término Violencia de Género como el equivalente a Violencia contra las Mujeres, tal como fue asumido por la Organización de las Naciones Unidas (1993) donde quedó definida como: "Cualquier acto de violencia basado en la pertenencia al sexo femenino que tenga o pueda tener como resultado un daño o sufrimiento físico, sexual o sicológico para la mujer, así como las amenazas de tales actos, la coacción o la privación arbitraria de la libertad, tanto si se producen en la vida pública como en la vida privada"[2].

Los elementos más importantes puestos de manifiesto en esta definición están relacionados con todas aquellas formas en la que se impone y persiste una relación de poder donde se incrementa la subordinación de las mujeres al género masculino develando los diversos daños que pueden ocasionarse a las mujeres, desde limitaciones en el ejercicio de su ciudadanía hasta la muerte. Pese a esta declaración, aún es un obstáculo para el abordaje del problema el significado que otorga la propia comunidad a la Violencia de Género, ese significado funda la construcción de las subjetividades, también conocido como imaginario social, y la implicación que supone este constructo en las prácticas sociales.

Desde la acepción de Fernández, M. y de Brassi, J (1993) "En el término imaginario social, lo imaginario remite a otro orden de sentido: ya no como imagen de, sino como capacidad 
imaginante, como invención o creación incesante social-histórica-psíquica, de figuras, formas, imágenes, en síntesis, producción de significaciones colectivas"[3]. Siendo el imaginario colectivo los significantes que la gente construye sobre situaciones vivenciales, en este caso la violencia de género, se hace indispensable considerar como estrategia fundamental en la prevención de la Violencia de Género, el empoderamiento, el cual está referido al reforzamiento de las habilidades y potencialidades que poseen las personas para tomar el control de sus vidas, identificar los problemas y/o necesidades que les afectan, tomar parte en la solución de los mismos, participar en la toma las decisiones y ganar autonomía.

En cuanto a su utilización en el proceso de lograr la equidad de género e igualdad de oportunidades, la Organización Panamericana de la Salud (2005) alude "otros no pueden darle poder a las mujeres, sólo las mujeres pueden empoderarse a sí mismas. Sin embargo, las instituciones pueden apoyar los procesos de empoderamiento tanto en el plano individual como en el colectivo"[4].

En consecuencia, uno de los intereses perseguidos a lo largo de esta investigación es: Rastrear las subjetividades construidas por mujeres y hombres sobre la Violencia de Género a fin de promover acciones preventivas, sostenibles y eficientes que contribuyan a elevar la calidad de vida de las mujeres.

\section{METODOLOGÍA}

El presente estudio obedece a una investigación con enfoque de género, abordada desde la modalidad cualitativa ya que toma en cuenta las construcciones subjetivas y concepciones de lo femenino y lo masculino para dar explicación a lo que las(os) participantes consideran sobre la Violencia de Género. La selección de los participantes fue intencional y el grupo estuvo constituido por un total de doce participantes de los cuales siete eran mujeres y cinco eran hombres que formaban parte de la comunidad El Rincón, municipio Naguanagua, estado Carabobo-Venezuela. La estrategia utilizada para la convocatoria de las(os) participantes incluyó el envío de comunicaciones, reuniones con líderes comunitarios u otros miembros de la comunidad para explicar la intencionalidad del estudio, así como la solicitud del consentimiento informado a las(os) participantes interesadas(os) en formar parte de la discusión, caracterizándose por ser una participación voluntaria, libre y espontánea.

La técnica utilizada para la recolección de los datos fue la observación directa lo que resultó favorable para describir las características de la comunidad, reconocer algunas particularidades socio demográficas y representar comportamientos o actitudes del grupo humano sujeto de este estudio. De igual manera, fue utilizada la técnica grupo focal de discusión lo que ameritó la elaboración de preguntas generadoras para profundizar en el tema de interés. La interpretación de los datos fue inductiva integrando para ello los planteamientos de Foucault en Rodríguez, R (1999) con respecto a: "el análisis del discurso, la genealogía de la identidad de género y el análisis de las relaciones de poder entre los sexos"[5]. Para la organización y sistematización de la información fue utilizado el software computacional Atlas ti 6.2 (versión Demo) facilitándose la construcción de categorías y la presentación de la relación de categorías en mapas de estructuras. Al respecto, Martínez, M (2004) sostiene "El mejor modo de comenzar la estructuración es seguir el proceso de integración de categorías menores o más especificas en categorías más generales y comprensivas. La estructura puede considerarse como una gran categoría más amplia más detallada y más compleja"[6]. 


\section{RESULTADOS Y DISCUSIÓN}

El acercamiento con los miembros de la comunidad, abrió paso a la observación directa, considerada en este caso como la primera fase del proceso investigativo, implicó la documentación, la compresión de la vida local y el establecimiento de un clima de confianza que fue logrado a medida que se explicaba a los miembros de la comunidad la importancia de escuchar la vivencia y las concepciones de este grupo humano acerca de la Violencia de Género.

En cuanto a las características físicas de la comunidad El Rincón, podemos indicar que se encuentra ubicada en el municipio Naguanagua del estado Carabobo, tiene una población de 988 habitantes aproximadamente; limita por el norte con la Urbanización Tazajal por el sur Urbanización Mañongo, al este de la comunidad se encuentra el Cerro El Trigal y en el oeste Autopista Valencia - Puerto Cabello, disponen de servicios públicos apreciándose deficiencias en el suministro de luz, agua y recolección de basura.

Para el momento de la investigación, el funcionamiento comunitario se encontraba en un proceso de reestructuración, efectuando las reuniones preliminares para conformar el Consejo Comunal, pese a ello, sus miembros mostraban participación activa, cooperación y asesoramiento por parte de líderes naturales (mujeres en su mayoría) que colaboraban con las(os) líderes emergentes que conducían la consolidación de la nueva estructura organizativa. Al no observarse en el contexto comunitario jerarquías de género, se decidió documentar la experiencia de mujeres y varones para enriquecer el tema de interés, un aspecto favorecedor es que las(os) participantes mostraron sentirse cómodas (os) y con la suficiente libertad para discutir acerca de sus vivencias y concepciones.

El grupo humano sujeto de este estudio puede describirse como personas que comparten intereses comunes, valores, costumbres y conviven en una misma localidad. En términos de escolaridad es similar el nivel educativo en ambos grupos, con excepción de una mujer (adulta mayor) que no completó la educación primaria; las mujeres y los hombres participantes en su totalidad eran madres y padres de familia y en cuanto a la situación conyugal la mayoría eran casadas(os); una divorciada; un divorciado y una viuda. En atención a la ocupación, se aprecia que cuatro de las mujeres participantes eran amas de casa, la ocupación laboral de las otras tres mujeres; fuera de tener la responsabilidad de los trabajos en el hogar y crianza de los hijos, eran trabajo doméstico en casa de familia y la venta de comida; en el caso de los cinco varones participantes la ocupación giraba en torno a la construcción, el comercio informal y la venta de comida los fines de semana; todos los hombres se describieron como colaboradores en los quehaceres del hogar.

Las observaciones y exploraciones previas acerca de la vida y quehacer de las(os) participantes propició el acercamiento entre el grupo y las investigadoras. Surgiendo, espontánea y naturalmente en la esfera familiar: los cuadros de sobrecarga en algunas mujeres (doble jornada laboral), abuela esclava, infravaloración del trabajo realizado dentro del hogar y posiciones de privilegio en los hombres, puesto que sus participaciones en los asuntos domésticos y en la crianza de los hijos eran eventuales, a pesar de que ellos en un principio se describían a sí mismos como colaboradores.

Todo esto resulta difícil de distinguir por el grupo y en particular por las mujeres participantes, ya que no existe conciencia de esta asimetría. Por ello, cuando profundizamos en el discurso, se observa a lo largo de la investigación, aceptación en lo referido al control y la dominación del hombre sobre la mujer, como la conducta apropiada y normal, desplegándose de esta manera algunos de los rasgos que caracterizan nuestra cultura 
patriarcal. A continuación registros de los intercambios realizados con tres de los varones participantes:

Nosotros los hombres lo que hacemos a veces es gritar al muchacho... por ejemplo: ¡Que yo estoy trabajando mucho y tú no te portas bien y tú mamá se pone fastidiosa!... Cuando el hombre llega estresado del trabajo y los muchachos están todo el tiempo: ¡Papá mi mamá me pegó! Entonces uno les dice: ;Te vas al cuarto sin comer! (Hombre)

La mujer es el policía que está prácticamente todo el día en la casa, el hombre es el que pone el ejemplo en la casa, cuando a ella no puede controlar la cosa. (Hombre)

Es que el hombre siempre está cansado porque es el que trabaja en la calle... (Hombre)

En referencia a lo anteriormente expuesto, conviene señalar a Huggins, M. (2005) quien sostiene "Mientras más tradicional sea la estructura de roles y los estereotipos de lo que significa ser masculino/hombre o femenina/mujer, habrá mayor probabilidad de que ambos sean construidos desde y para un espacio intersubjetivo violento, con papeles fijos dentro de la estructura jerárquica: dominantes, fuertes, violentos, ellos; y dominadas, débiles y violentadas, ellas" [7].

La segunda fase de la investigación comprendió: el acopio de los relatos con la suficiente discusión de los temas tratados en el grupo focal, los cuales fueron archivados en documentos de voz y luego transcritos para su posterior exanimación, reexaminación y el análisis de la información recogida. De este proceso irrumpieron un conjunto de unidades temáticas que cedió a la posibilidad de comprender y dar sentido a las concepciones de mujeres y hombres de la comunidad El Rincón en relación a la Violencia de Género. Seguidamente se presentan a detalle las categorías obtenidas y su estructuración posterior al análisis: Dilucidaciones de la Violencia de Género, Doble victimización de la Mujer, Mujer como objeto de deseo sexual y Recomendaciones para la Prevención de la Violencia de Género (VG) (Ver imagen 1) 


\section{Imagen 1}

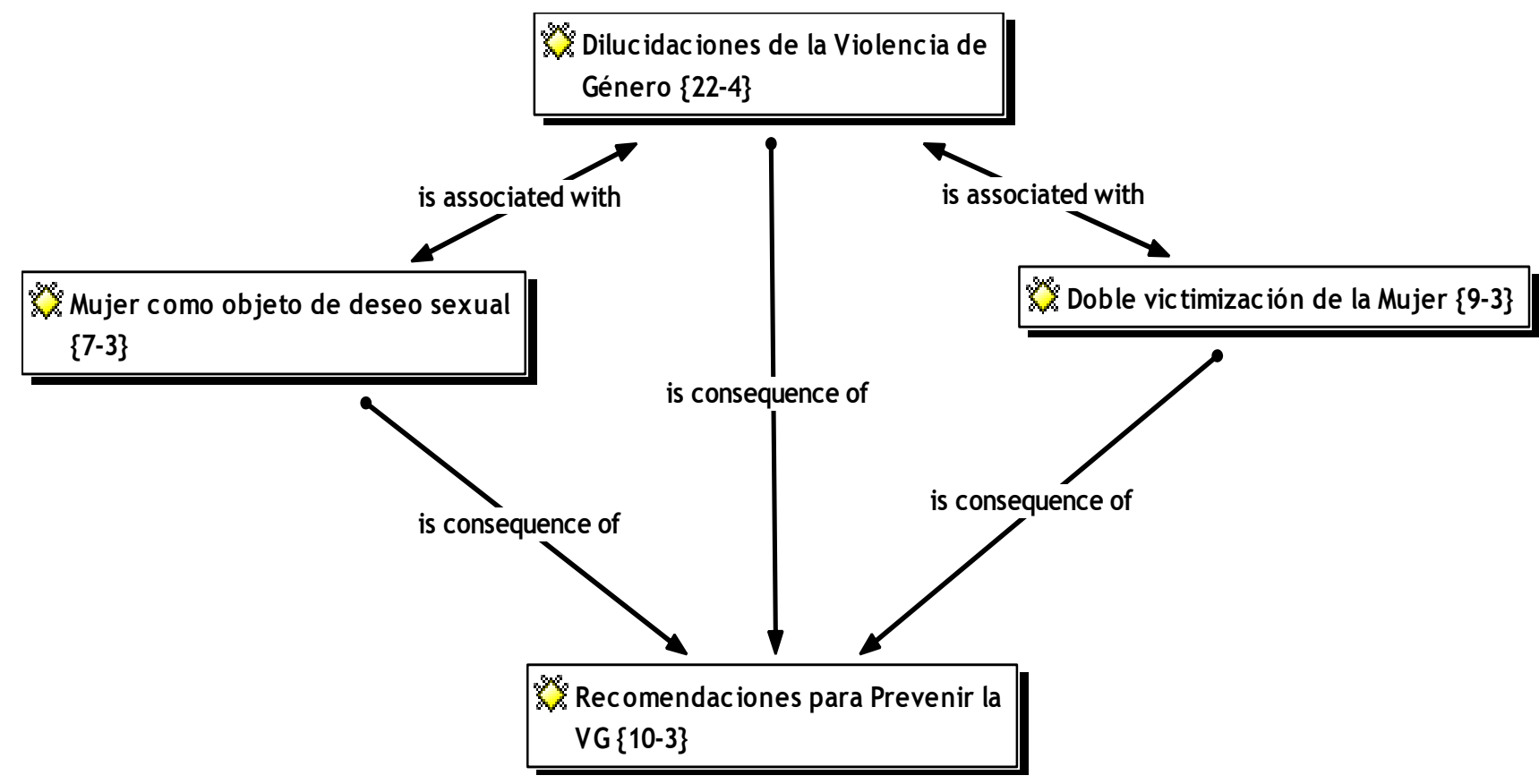

Primera Red Estructural

Basándonos en los testimonios de las(os) participantes, el eje central para el análisis del modelo estructural es "Dilucidaciones de la Violencia de Género" esta categoría está referida a los juicios de las(os) participantes en relación a lo que consideran Violencia de Género y todas las enunciaciones obedecen a las experiencias vividas por el grupo a lo largo de sus vidas:

1:1 "La violencia de género se hace cuando uno verbalmente agrede a una persona" (2:2) (Hombre),

1:2 "Cuando te dicen bruta delante de otra persona... eso es una forma de violencia a la Mujer" (2:2) (Mujer),

1:3 "Cuando la mujer se viste y se arregla... El hombre le dice: ¿Para dónde vas tú así? Anda a quitarte eso" (38:38) (Hombre),

1:5 "Por ejemplo cuando uno tiene amigas y está hablando y riéndose con ellas... Los hombres están con la desconfianza y siempre preguntan todo:¿Por qué hablaron en voz baja?, ¿Por qué se rieron?” (45:45) (Mujer),

1:6 "Yo conozco hombres que después que se casan le quitan a las mujeres las amistades y hasta le prohíben a la mujer ir a visitar a la mamá..."(48:48) (Hombre).

1:8 "Yo siempre bajé la cabeza... tuve una pareja agresiva... siempre estuve al lado de él para mantener la familia... Cuando había maltrato me sentía mal por mí... (60:60) (Mujer),

1:9 "Siempre él las ganaba todas... cuando él hablaba me dejaba a mi sin palabra... todo lo que yo pensaba, me lo reservaba..."(65:65) (Mujer), 
1:10 "Me sentía como nada, él decidía todo, nunca hice nada por mí, lo aguanté porque yo pensaba que todos los hijos que tuve necesitaban un padre, para poderse criar bien... sentía mucho rencor" (68:68) (Mujer),

1:11 "Yo trabajaba en la casa haciendo arepa, todavía hago venta de todos tipos... yo aportaba mucho... a veces más que él... A mí me tocaba pagar transporte, pagar los libros, sí me ayuda pero cuando él estaba molesto por algo no me da los reales..." (73:73) (Mujer),

1:12 "Lo que pasa es que antes, las madres enseñaban a las hijas que tenían que mantenerse con los maridos... No se podían separar, no lo podían dejar y tenían que aguantarse todo..." (76:76) (Mujer)

1:13 "Es verdad... Mi mamá siempre me decía: Sé fiel y por eso soporté!" (79:79) (Mujer)

1:14 "Tuve que dejar los estudios, iba por el quinto semestre, pero por la desconfianza y el orgullo macho me tuve que retirar... Dejé los estudios para quitarme ese sobresalto, el temor que tenía todo el tiempo, estaba en clase y era angustiada..." (62:63) (Mujer).

1:15 "Yo conozco a alguien... es mi hermana... ella está estudiando ahorita, ella está un poco mayorcita, los hijos de ellos dos han crecido, empezó a estudiar porque quiere superarse... Ahora el esposo dice: No me gusta que vaya a estudiar... Entonces ahora entre ellos siempre hay un roce, todo el tiempo una pelea... Hay siempre esa desconfianza... él busca la discusión como para que ella deje los estudios y se quede en la casa..." (41:41) (Hombre),

1:4 "Ahorita aquí en nuestra comunidad se está viendo un caso de maltrato físico presuntamente esta es la segunda vez que la persona reincide en el maltrato a la mujer, no se saben cuáles son las causas que lo llevan a que él le caiga a golpes a la esposa" (12:12) (Mujer),

1:16 "Ese es un ejemplo de maltrato... Es una discusión que ocurre internamente, por lo general ninguna familia se mete... Uno oye y se queda callado... Claro este problema que sucedió muchas veces hasta que la comunidad se tuvo que meter $y$ apoyar..." (13:13) (Hombre).

Tal como se ha observado en las narraciones, las principales formas de Violencia de Género reconocidas o vividas por el grupo humano en estudio fueron: Violencia verbal, Violencia psicológica, Violencia económica y Violencia física; en los relatos se demostraron interferencias por parte de los agresores en lo relacionado con: la autonomía, la autoimagen, la preparación académica, la productividad, el otorgamiento del dinero, la socialización con amigos y con la propia familia.

Todas las acciones antes referidas se corresponden con lo considerado por Bonino, L (2002) como "Modelo social de la masculinidad tradicional hegemónica"[8] apoyadas en las prácticas de dominación masculina causando en las victimas problemas de convivencia, trastornos de salud, dependencia, frustración y sufrimiento. Llama poderosamente la atención, específicamente en referencia al caso de la mujer que fue víctima de la Violencia Física por parte de la pareja (hombre), que en las declaraciones dadas por algunas(os) participantes es notoriamente identificable la naturalización del problema, minimizando la 
gravedad de este atropello. De igual manera, las afirmaciones realizadas colocan a la mujer en una posición de desventaja frente a las situaciones de agresión, ya que la comunidad no percibe, ni reconoce la Violencia de Género como un problema público y de obligatoria intervención, el silencio es la primera reacción, luego consta en los relatos la mediación porque la agredida busca apoyo. A continuación otras manifestaciones del grupo en torno al mismo caso:

\section{1:17 "Cuando ocurrió la primera golpiza... no se toco el tema” (13:13) (Mujer),}

1:18 "Hubo que recoger firmas y hacer denuncia para que no ocurriera el maltrato otra vez" (13:13) (Hombre).

1:19 "Claro es algo que ya no ocurre dentro de la familia... como un asunto familiar... sino es algo que se salió porque la mujer busco la ayuda de la comunidad..." (15:15) (Mujer),

Observaciones: La actitud del grupo (mujeres y hombres) fue en este punto mostrar respeto por lo que ellos consideran la intimidad familiar.

Por otra parte, surge en el discurso como un hecho aislado la agresión física contra el varón perpetrada en este caso por su pareja (mujer). Vale la pena enfatizar, que el maltrato contra el varón no puede ser comparable ni en magnitud ni en consecuencias para la salud, con la Violencia que ejercen los hombres hacia las mujeres. Es por ello, que cuando se habla de Violencia de Género se hace referencia única y exclusivamente a las mujeres. Respectivamente, Bihler, M. (2005) asegura "La desigualdad de poder entre mujeres y hombres es la base para la violencia de género, y este es el punto donde la mujer se encuentra en desventaja, dando lugar a una gran mayoría de mujeres víctimas de la Violencia de Género." [9]. Asimismo, una investigación realizada recientemente por Ansara e Hindin (2010) sobre las consecuencias psicosociales de la violencia de pareja en hombres y mujeres en Canadá reveló en 676 mujeres y 455 hombres que informaron haber sufrido violencia física o sexual por parte de un(a) actual o ex pareja que "las personas que sufren los cuadros más severos y crónicos de patrones de violencia y control reportan el impacto más negativo. Estos resultados fueron especialmente pronunciados por las mujeres que experimentaron el patrón más crónico de abuso y de control documentados en el estudio. Las consecuencias psicosociales también fueron mayores para las mujeres que para los hombres con experiencias similares de maltrato" [10].

Sin embargo ${ }_{2}$ tanto el hecho violento, como el incumplimiento del ideal masculino impuesto por los estereotipos de género correspondientes a los valores del modelo de género tradicional suponen el desencadenamiento de procesos que conllevan a la aparición de riesgos o problemas de salud en la persona que es agredida, por tanto su detección incluye la correspondiente asistencia del caso. Una de las observaciones realizadas durante la discusión del tema, muestra en el grupo la expectativa del ideal masculino tradicional; esto ocurre al surgir comentarios en los que se espera una reacción que ubique al hombre en un plano de superioridad con respecto a la pareja (mujer), exhibiéndose a su vez, una violencia simbólica en la que se establece la desvalorización, subordinación y discriminación de las mujeres. Obsérvese el relato y las anotaciones realizadas:

1:21 "Hay la otra parte que no se ve mucho pero yo tengo un amigo que la mujer es la agresiva yo lo he visto que llega golpeado sin motivo, pero él no puede hacer nada... ¿Quien lo ayuda a él? Lo que puede conseguir es quedar en ridículo” (32:32) (Hombre), 
1:22 Observaciones: Algunos miembros del grupo (mujeres y hombres) se rieron e hicieron comentarios acerca de la debilidad del hombre.

A su vez la categoría "Dilucidaciones de la Violencia de Género" está asociada con la categoría "Mujer como objeto de deseo sexual" que fue identificada por el grupo como otro tipo de Violencia. Esta se refiere a la manera como la mujer es colocada en una situación de pasividad frente al posicionamiento del hombre que idealiza su potencia sexual como rasgo de masculinidad. Efectivamente, lo señalado por las(os) participantes corresponde a lo que Galtung, J. (2003) define como los medios por los que se ejerce la violencia cultural, entre ellos destacan: "la religión, la ideología, el lenguaje, el arte, los medios de comunicación, etc."[11].

A continuación los aportes de las personas que formaron parte del grupo focal:

1:23 "Una agresión contra la mujer, hoy en día, es la letra de las canciones de Reggaetón, porque humillan a las mujeres, la baja de categoría los cantantes con todos esos mensajes la ponen por el piso..." (80:80) (Hombre),

1:26 "Así como se expresan en las canciones se expresan los hombres de las mujeres..." (81:81) (Hombre), 1:25 "Lo que pasa es que antes eran mensajes subliminales ahora son explícitos porque el mensaje viene clarito en las canciones de moda: El llamado ahora es para el sexo desatado, la violencia y la droga..." (82:82) (Mujer),

1:27 "Creo que lo que está sucediendo... siguiendo con el tema de la música, es que definitivamente se usa a la mujer como un producto..." (84:84) (Mujer),

1:28 "La mujer aparece prácticamente sin ropa en los videos, se muestra deseosa de sexo, se degrada como persona y solamente se beneficia el hombre que es el que vende el producto... Entonces ¿qué valor tiene la mujer ahí? Nada... La mujer la usan para vender cualquier cosa y eso es violencia contra la Mujer." (85:85) (Hombre).

Luego de las anteriores declaraciones es conveniente hacer mención de un material preparado por el Instituto de la Mujer de España, La publicidad y la salud de las mujeres (2010). Allí se considera que "El imaginario social se transmite mediante mensajes constantes que son recibidos e incorporados por las personas, y que tienen especial impacto en la construcción de la subjetividad a través de: la socialización y el desarrollo psíquico de cada cual, que incorpora como propios los modelos ideales de masculinidad y feminidad"[12].

Desde este punto de vista resulta viable mostrar la relación existente entre comunicación y la construcción del género en cada persona, es así como la discusión surgida en el grupo focal trae a colación aspectos bastante interesantes para ser analizados. En primer lugar ciertamente, existe una difusión a través de los medios de comunicación de mensajes que conectan el cuerpo de la mujer con el placer solo para complementar y colmar los deseos del hombre hecho que es muy bien reconocido por la comunidad. En oposición, emerge del discurso de las(os) participantes, un contenido que deja en evidencia la expectativa del comportamiento de la mujer acorde con los principios del modelo de género tradicional donde se destacan la sumisión, la subordinación y la pasividad. Apoyando lo antes planteado en un material elaborado por el Centro Nacional de Equidad de Género y Salud Reproductiva (2005) se menciona que: "Los estereotipos de género no necesariamente se 
cumplen, pero sirven de patrón de medida que influye en el juicio, en la valoración social, en la propia imagen y autoestima"[13].

En atención a lo expuesto seguidamente se presentan otros extractos de las(os) participantes en la discusión grupal:

1:29 "Muchas veces las mujeres se prestan para eso regalándose a los hombres porque el valor de guardarse para un hombre se ha perdido... Ahora las mujeres andan buscando macho... pero todo eso puede ser producto de lo que se está hablando" (81:81) (Hombre),

1:30 "Aquí hay varias mujeres que ponen esas canciones y cuando tú volteas y las ves están bebiendo ron igualito como si fueran hombres" (83:83) (Mujer).

De la categoría "Dilucidaciones de la Violencia de Género" surge una segunda asociación denominada "Doble victimización de la Mujer" referida a la construcción subjetiva en la que se le confiere a la mujer toda la responsabilidad y culpa por los sucesos ocurridos en su entorno.

1:31"Eso es un problema porque tú te encuentras atado de pies y manos porque no sabes a quien vas a apoyar tú..." (18:18) (Mujer),

1:32 “Eso es obvio, tienes que apoyar a la víctima no al agresor.” (20:20) (Mujer),

1:33“Hay que averiguar primero: ¿Qué problema tiene esa persona? ¿Por qué agrede? ¿Qué hace la otra persona para que la agredan?... (21:21) (Mujer),

1:34 "Yo creo que no hay motivos para llegar a la agresión, yo no veo como una excusa: mi esposo me pegó porque tenía que hacerlo" (22:22) (Hombre),

1:35 "Yo lo que creo, es que: Si esa persona recibe golpe una y otra vez ¿Por qué regresan y vuelven a vivir juntos? Tiene que haber algún problema" (23:23) (Mujer),

1:36 "La comunidad ha tenido que salir a apoyar a la mujer agredida y al poco tiempo regresan otra vez... Entonces eso afecta a la comunidad... "Ha llegado un momento en que la gente está molesta y dice: "A ella le gusta" "A mí que no me llamen a firmar otra vez por lo mismo" y "Podré ver que se están matando y no nos vamos a meter" Esos son comentarios de los vecinos..." (25:25) (Mujer),

1:37 "Hay gente que aprende a vivir muy sabroso en ese ámbito y de alguna manera se establece como un estilo de vida de esa manera... hay que darse cuenta de varias cosas el que hace sufrir está preocupado porque lo está haciendo y la persona que es víctima muchas veces busca la forma de que la situación de violencia se repita..." (27:27) (Mujer),

1:39"Ese muchacho es débil porque él no se puede aguantar que ella lo busque y en vez de él darle la espalda y no continuar con esa situación siguen juntándose y siguen las golpizas" (58:58) (Mujer).

Observaciones: En contraposición a todas las afirmaciones donde se culpa a la mujer de la situación de violencia surgieron dos intervenciones de desacuerdo que el grupo escuchó para después justificar con mayor vehemencia sus perspectivas. 
Cabe destacar que la discusión conllevó a una dinámica donde algunas(os) participantes revelaron a través del discurso subjetividades en las que consienten la dominación y la agresividad desplegándose de esta manera el poder patriarcal en el cual históricamente le fue otorgado al varón el derecho de controlar, disponer y castigar libremente a su esposa e hijos ya que eran considerados de su propiedad. Las actitudes y el discurso de las personas participantes en el grupo focal, crean un marco que de alguna manera normaliza la violencia en la relación de pareja, ese hecho concreto y refiriéndonos nuevamente a Galtung es lo que se considera violencia cultural "expresada desde infinidad de medios, y que cumple la función de legitimar la violencia directa y estructural, así como de inhibir o reprimir la respuesta de quienes la sufren, y ofrecer justificaciones para que los seres humanos, a diferencia del resto de especies, se destruyan mutuamente y sean recompensados incluso por hacerlo"[11].

Finalmente, en el proceso de estructuración del conocimiento la categoría "Recomendaciones para la Prevención de la VG" es presentada como la consecuencia del resto de las categorías analizadas, y constituye un valioso aporte para el desarrollo de acciones preventivas, ya que estima las particularidades socioculturales en los que se desarrolla la vida de las mujeres. Tal como se mostrará a continuación, la categoría engloba las sugerencias dadas por las(os) participantes en lo que corresponde a la prevención del problema:

1:40 "Hablar con las mujeres cuando están jovencitas, decirles que si se consiguen una pareja se quieran y se respeten... pero si pasa en algún momento lo mismo que pasaba yo... que no lo aguanten ... que se alejen..." (93:93) (Mujer),

1:41 "Consolidar los valores en el hogar, respetarlos y enseñarlos a respetar, establecer normas y líneas para la crianza de las niñas y los niños también, eso les ayuda a tener su personalidad y a una persona segura no la agrede nadie" (94:94) (Mujer),

1:42 "Lo que se enseñe en la casa sirve para prevenir la violencia de la mujer... A mi mis hijos me ven barriendo, cocinando y fregando platos... Viendo se les enseña" (95:95) (Hombre),

1:43 "Una forma es diciéndoles a las madres que hablen sobre todo con los varones... en mi caso los varones presenciaron el maltrato que me hacia el papá y yo siempre hablaba con ellos y les decía ustedes tienen que ayudar a la mujer con la que hagan su vida..."(96:96) (Mujer),

1:44 "A las hembras mi consejo era enamórense pero no se dejen de nadie... el hombre que no sirva, que las esclavice o que las maltrate... déjenlo porque van a tener una vida infeliz." (97:97) (Mujer),

1:45 "Es muy importante la gente que vive en la comunidad de uno, yo pude haberme descarriado... en mi casa también había mucho problema entre mi papá y mi mamá pero cuando yo iba a recoger el monte en las casas de los vecinos me hablaban... y daban concejo de cómo debíamos comportarnos" (98:98) (Hombre),

1:46 "El lineamiento que sí debemos tener claro es plantearle siempre a las dos partes que necesitan ayuda profesional... Hacerles entender que tienen un problema y que necesitan ayuda..." (99:99) (Mujer), 
1:47 “Hablar de la sana convivencia dentro de la comunidad...” (100:100) (Mujer),

1:48 “Enseñar a la gente... hace falta talleres” (101:101) (Mujer),

1:49 “La comunidad influye bastante..." (102:102) (Hombre).

En los discursos, es evidenciada la imperiosa necesidad que tiene el grupo de clarificar las concepciones sobre la Violencia de Género, aspecto que no fue percibido por los participantes, siendo entonces vital para la prevención del problema la creación de espacios de reflexión y discusión donde se cuestionen los estereotipos de género tradicionales y se promueva las relaciones de igualdad entre seres humanos; sin duda alguna, estas acciones garantizan el desarrollo de las comunidades y elevan la calidad de vida de sus miembros. En las recomendaciones dadas, observamos que el grupo demanda la inclusión de mujeres y varones en el proceso formativo, emerge como principal protagonista la educación dentro del hogar con la transmisión de valores tales como: (respeto, solidaridad y justicia), el modelaje de funciones donde mujeres y hombres asuman por igual los roles y responsabilidades dentro del hogar, así como la preparación de los miembros de la comunidad para que actúen como factor de protección.

\section{A MODO DE CONCLUSIÓN}

Entendiendo el compromiso de la atención primaria, en la prevención y detección temprana de los casos, consideramos trascendental haber rastreado los significados que otorga la comunidad a la Violencia de Género puesto que constituyó una fuente inagotable que nos acercó a una realidad en la que encontramos, no solamente los efectos notorios de la violencia en sus víctimas, sino también, aleccionamientos sobre las distintas formas en que se presenta la violencia de género en la comunidad.

Adicionalmente, la comprensión de las subjetividades construidas por mujeres y hombres sobre la Violencia de Género permitieron comprobar rasgos en el decir y el quehacer social que posiciona a las(os) participantes en la práctica de valores del modelo de género tradicional. Asimismo, fue evidente la necesidad de recibir suficiente y acertada información sobre Violencia de Género, utilizando los resultados de este estudio, como un elemento que permita a las(os) participantes reflexionar sobre sus propias concepciones y así promover en ellas(os) la voluntad explícita del cambio.

Para finalizar, es importante considerar las propuestas dadas por el grupo humano sujeto de este estudio para la Prevención de la Violencia de Género, ya que sugiere la inclusión de mujeres y varones en el proceso educativo, otorgándoles a la familia y a los miembros de la comunidad especiales responsabilidades durante la transmisión de valores. La actividad preventiva deberá incluir el impulso de una política institucional que promueva significativamente: el trabajo interdisciplinario, actividades de sensibilización comunitaria periódicas, información que provea a las personas de una actitud crítica para deconstruir los estereotipos de géneros tradicionales, contemplando, además, estrategias de seguimiento para evaluar la efectividad de las acciones realizadas.

\section{BIBLIOGRAFÍA}

1. Bonder, G. Género y Subjetividad (1998) Avatares de una Relación no Evidente. Programa Interdisciplinario de Estudios de Género -PIEG- Universidad de Chile.

2. Naciones Unidas (1993) Declaración sobre Eliminación de la Violencia contra la Mujer. Nueva York-U.S.A: Naciones Unidas. 
3. Fernández, Ana y de Brassi, Juan (1993) "Tiempo Histórico y Campo Grupal. Masas, grupos e instituciones". Nueva Visión, Buenos Aires.

4. Organización Panamericana de la Salud (OPS) (2005). Proyecto de política de la OPS en materia de igualdad de género. En: http://www.paho.org/Spanish/AD/DPC/VP/rimsa14-inf4-s.pdf

5. Rodríguez, Rosa (1999) Foucault y la Genealogía de los sexos. Barcelona: Anthropos, Universidad Autónoma Metropolitana.

6. Martínez, Miguel (2004) Ciencia y arte en la Metodología Cualitativa. Editorial Trillas. México.

7. Huggins, M. (2005) Género, políticas públicas y promoción de la calidad de vida. Instituto Latinoamericano de Investigaciones Sociales (Ildis), Venezuela.

8. Bonino, L (2002) Masculinidad, salud y sistema sanitario -El caso de la Violencia Masculina- Artículo publicado en «Seminario sobre Mainstreaming de género en las políticas de salud de Europa» Instituto de la Mujer-España OMS.

9. Marion Bihler (2005) Gender and Citizen security. Published by Project for the Promotion of Gender Policies / GTZ - Nicaragua. ISBN: 99924-0-377-2 (Vol. 1) in: http://www.gtzgenero.org.ni/ publicacion/1130869381 basictext.pdf

10. Ansara, D. Hindin, M. Psychosocial Consequences of Intimate Partner Violence for Women and Men in Canada. Interpers Violence May 2010, doi: $10.1177 / 0886260510370600$

11. Galtung, Johan (2003) Tras la violencia, 3R: reconstrucción, reconciliación, resolución. Afrontando los efectos visibles e invisibles de la guerra y la violencia. Gernika: Bakeaz / Gernika Gogoratuz.

12. Instituto de la Mujer de España (2010) "La publicidad y la salud de las mujeres, Análisis y recomendaciones". Paper especialmente preparado para el seminario La integración del enfoque de género en el abordaje de la salud: Aportes conceptuales y metodológicos para fortalecer la calidad y la equidad", Cátedra UNESCO Mujer Ciencia y Tecnología - Facultad Latinoamericana de Ciencias Sociales (FLACSO) Sede Argentina.

13. Centro Nacional de Equidad de Género y Salud Reproductiva (2005) Género y Salud. Una introducción para tomadores de decisiones. Secretaria de Salud GuadalajaraMéxico. 